\title{
Achilles Tendon Rupture Secondary to Kite String (Manja) Injury: A Rare Etiology seen in Two Cases
}

\author{
${ }^{1}$ Vaibhav Bagaria, ${ }^{2}$ Amit Nemade, ${ }^{3}$ Nilesh Joshi
}

\begin{abstract}
Achilles tendon is the strongest and the thickest tendon of the human body. Due to its superficial location and stress, it has to face due to loading, the tendon is susceptible to injuries and ruptures. This is aggravated due to the precarious blood supply in the region and also age-related changes that occur in the tendon. Of the specific causes age-related and steroid induced tendinosis and sports injuries are the commonest mechanism of injury. Kite playing is a very common sport in the Indian subcontinent, which involves the use of a string (manja) often coated with powdered glass to fly the kite. Many grievous injuries are reported due to its use especially in two wheeler riders. However, to the best of our knowledge a kite thread injury causing Achilles tendon tear due to direct impact has so far not been reported. In this case series, we describe two cases that had such injury and a brief note on specifics of management of this case is described.
\end{abstract}

Keywords: Achilles tendon rupture, Manja, Tendon repair.

How to cite this article: Bagaria V, Nemade A, Joshi N. Achilles Tendon Rupture Secondary to Kite String (Manja) Injury: A Rare Etiology seen in Two Cases. J Foot Ankle Surg (Asia-Pacific) 2015;2(2):90-93.

\section{Source of support: Nil}

Conflict of interest: None

\section{INTRODUCTION}

Achilles tendon injury can be acute or chronic. The commonest age group of presentation is in middle aged athletes or weekend warriors-a term used for people who indulge in sporting activities on occasional basis. Acute rupture resulting from direct trauma are reported rarely, the commonest reported cause being secondary to indirect force resulting from sudden loaded plantar flexion of the ankle on full-weightbearing, especially

\footnotetext{
${ }^{1}$ Joint Replacement and Sports Surgeon, ${ }^{2,3}$ Lecturer

${ }^{1}$ Department of Orthopedics, Sir HN Reliance Foundation Hospital, Mumbai, Maharashtra, India

${ }^{2}$ Department of Orthopedics, NKPSIMS and ORIGYN Clinic Nagpur, Maharashtra, India

${ }^{3}$ Department of Orthopedics, NKPSIMS, Nagpur, Maharashtra India
}

Corresponding Author: Vaibhav Bagaria, Joint Replacement and Sports Surgeon, Department of Orthopedics, Sir HN Reliance Foundation Hospital, Mumbai, Maharashtra, India Phone: 9552329993, e-mail: bagariavaibhav@gmail.com while running on an incline or jumping. The unusual direct injury as a result of the kite string during kite flying festival season is a very rare cause. These kite strings called as Manja (or Manjha) employed for kite flying in Indian subcontinent and Afghanistan are abrasive strings usually gummed, colored and coated with the powdered glass. The management of this injury apart from the routine repair of the tendon also involves additional steps especially in regards to the infection prevention and assessment of neurovascular status in acute cases. A case series of two unusual cases-one a delayed presentation and one acute presentation of similar injury are reported below.

\section{CASE REPORTS}

\section{Case 1}

A 38-year-old lady presented to our orthopedic outpatient department (OPD) with complaints of difficulty walking and climbing stairs which developed after she suffered an injury to ankle 5 months earlier. The mechanism of initial injury was narrated as a kite string colloquially called as manja getting entangled around her ankles while walking. Patient reported that the impact of manja resulted in an incised wound on posterior aspect of left ankle and anterior aspect of the right ankle. The primary care physician underestimated the wound to be superficial and just cleaned and dressed it. The wound subsequently healed primarily without any problem. On her first examination at our hospital, we found her walking without a significant heel push off. There was a transverse healed linear scar 3 to $4 \mathrm{~cm}$ above the heel (Fig. 1). A defect was clearly felt in the Achilles tendon and the proximal and distal stumps of the ruptured tendon were palpable with a separation of 3 to $4 \mathrm{~cm}$. The Thompson test, $\mathrm{O}^{\prime}$ Brien needle test, calf squeeze test and the sphygmomanometer test were positive. The plantar flexion was weak. A clinical diagnosis of ruptured Achilles tendon was made. Sensory and vascular examination of the foot was normal. Radiograph of the ankle did not reveal any bony abnormality. An ultrasound of the tendon confirmed the diagnosis of Achilles tendon tear with a separation of $3 \mathrm{~cm}$ between the torn ends.

In view of the old injury and the defect, a surgical reconstruction of the tendon was planned. In prone 


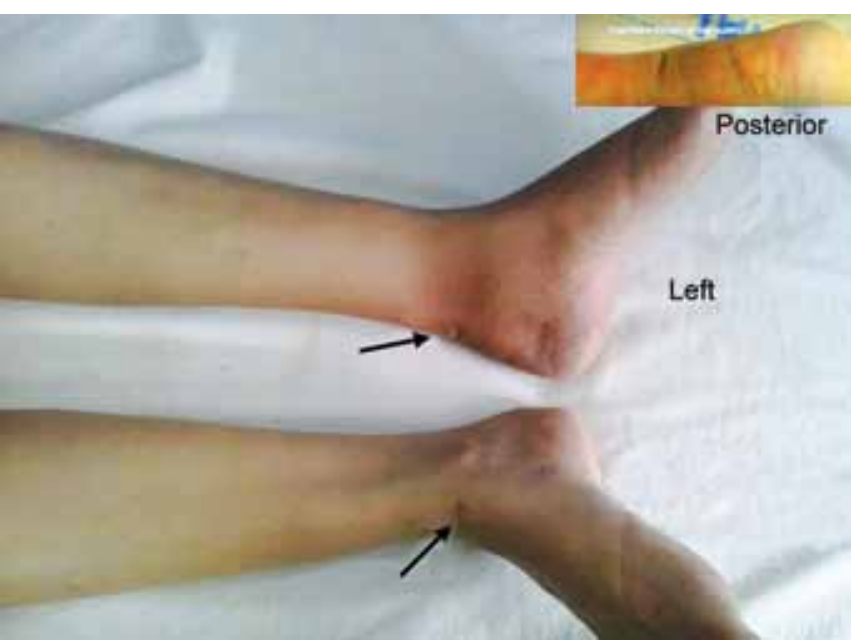

Fig. 1: Case 1: Clinical picture showing both feet with healed scar on posterior aspect of left ankle and anterior aspect of right ankle. In the inset, posterior extent of scar on left ankle is seen. Patient who had presented 3 months after the injury was disabled by inability to climb stairs or run

position, under spinal anesthesia and tourniquet control a curvilinear incision from lateral aspect of distal 1/3rd tendon crossing on to midline extending to upper calf was made. With sharp dissection keeping the skin flap as thick as possible the deep fascia was incised. The separation between the cut ends was clearly visible with some fibrous tissue intervening between them (Fig. 2). Considering the amount of defect and difficulty in end to end approximation, it was decided to do a Bosworth repair (Fig. 3). After freshening of the edges, a $2 \mathrm{~cm}$ central slip of the Gastrocnemius aponeurosis and Achilles tendon of $15 \mathrm{~cm}$ length was raised from proximal part, keeping the distal attachment intact. The slip was then routed transversely through the substance of the distal stump just above the insertion (Fig. 4). The tip was then passed through the proximal stump and the terminal part was fanned out to cover the defect and sutured with a non-absorbable suture material. The sural nerve and

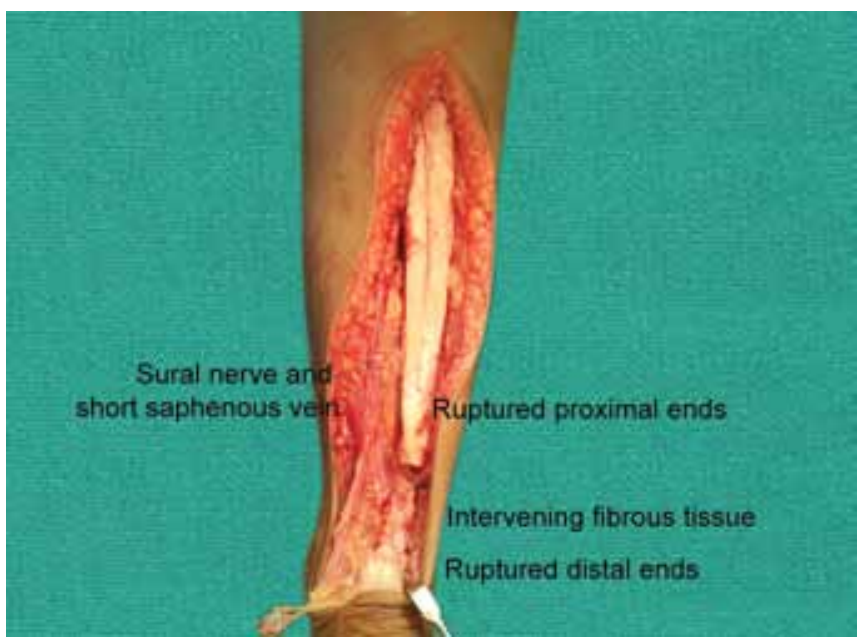

Fig. 2: Intraoperative picture showing the cut proximal and distal ends with intervening fibrous tissue

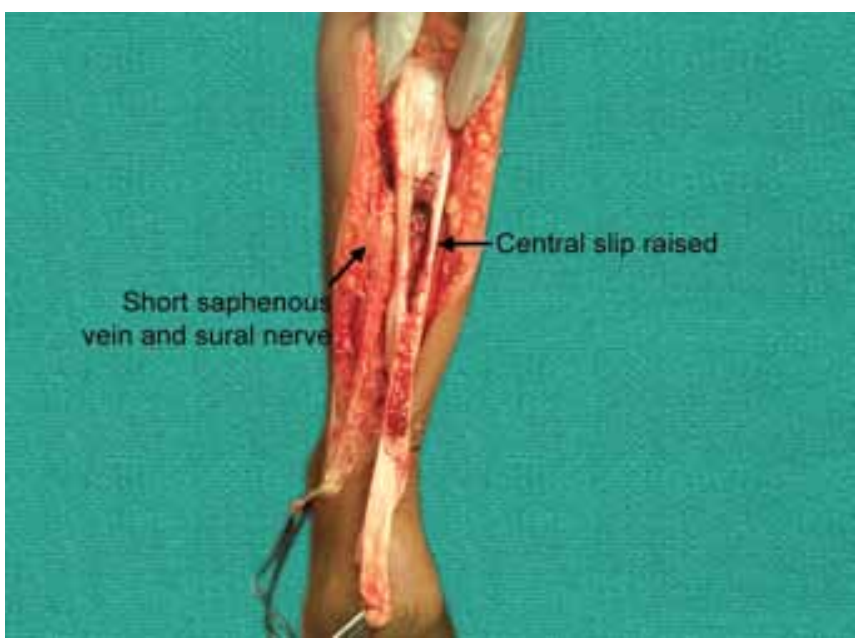

Fig. 3: Central slip raised from the proximal part of Achilles tendon and reflected distally to augment the repair

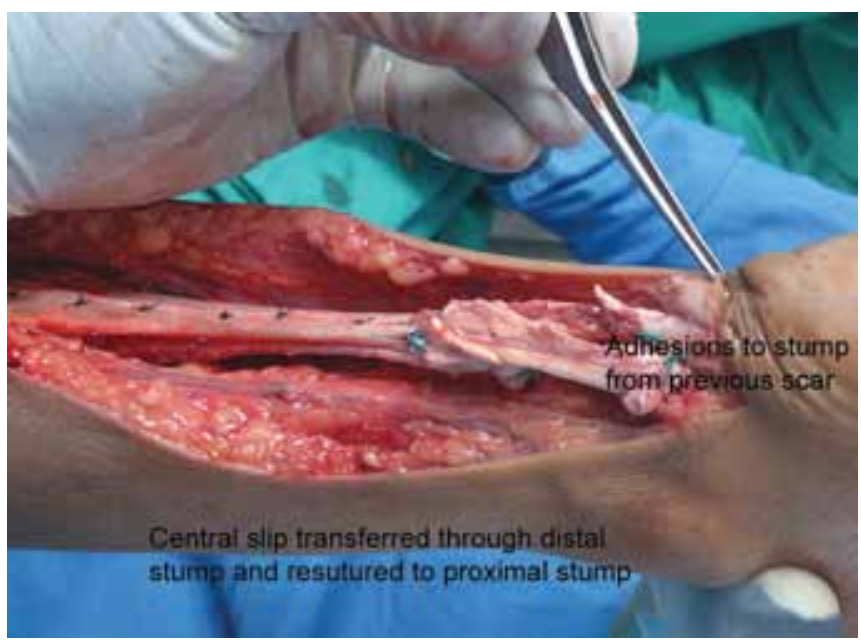

Fig. 4: Raised slip passed through the distal stump and sutured back to proximal stump

the short saphenous nerve were protected throughout the procedure. The defect from the harvest area was approximated.

The wound was then closed leaving the deep fascia open. An above knee cast with knee flexion and ankle in plantar flexion. The postoperative period was uneventful and the sutures were removed after 3 weeks and cast changed to below knee with ankle in slight plantar flexion. Meanwhile patient was advised to do static gastroc exercises and distal toe movements were encouraged. At the end of 6 weeks, below knee cast was removed. Ankle foot orthosis was given and stretching and strengthening of triceps surae was started along with toe touch weightbearing.

At the end of 18 months, patient can now walk fullweightbearing and has as reasonable plantar flexion power. Her active dorsiflexion and plantar flexion were good (Fig. 5). 


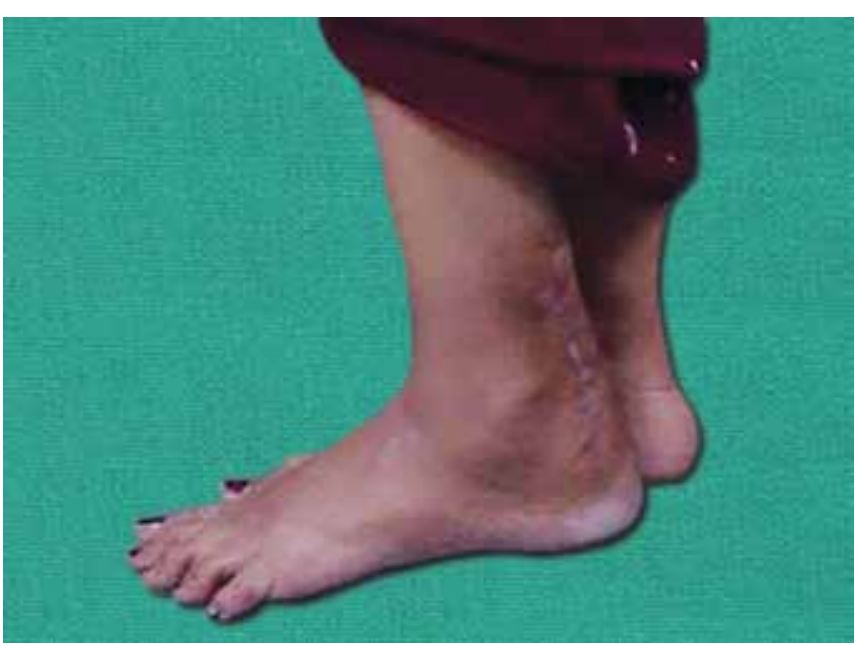

Fig. 5: Clinical picture showing an active plantar flexion at the end of 4 months postsurgery

\section{Case 2}

A 52 years old presented with an acute trauma to the posterior part of the ankle. The reported mechanism of injury was secondary to kite string getting entangled in her ankle while she was standing by the side of road and a motorbike whizzed past her. She presented with an open wound at the posterior aspect of ankle with a complete tear of the Achilles tendon (Fig. 6). The wound was immediately washed and dressed in the emergency room (ER) and patient underwent a primary repair of tibialis anterior (TA) in the operating room. A thorough wash along with tetanus prophylaxis and an antibiotic cover was ensued. The injury being fresh with clean cut tendon ends, a primary repair with Krackow type suture was performed. The entire surgery was carried out through the original wound which was a $5 \times 2 \mathrm{~cm}$ horizontal wound. During exploration, it was noticed that the posterior tibial artery and vein was

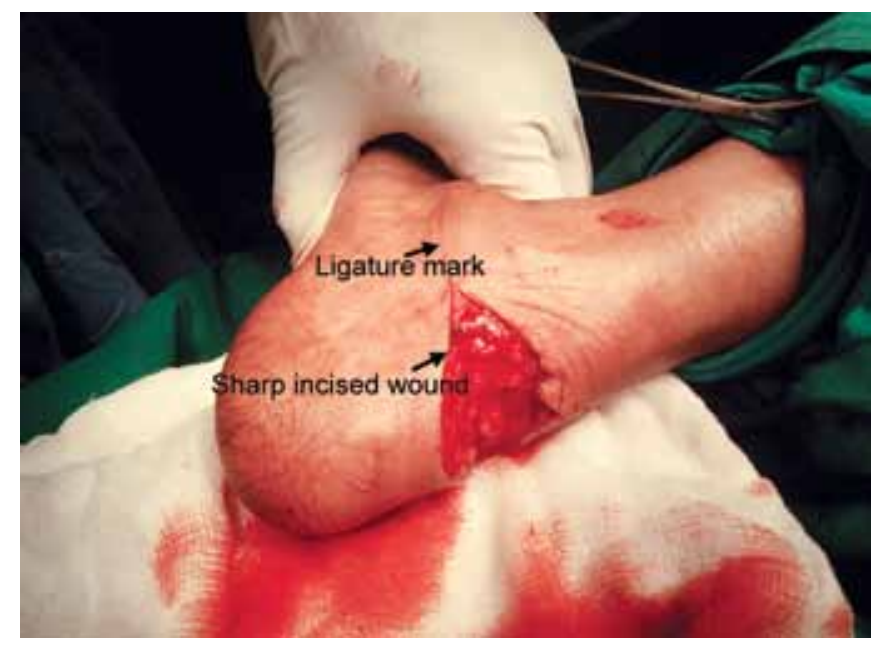

Fig. 6: Case 2: Injury seen primarily in the ER. Note a clean incised wound at the level of TA insertion. A tell-tale sign of the string injury extending anteriomedially can also be seen intact though the string had apparently been very close to severing it which could have been catastrophic (Fig. 7). Standard postoperative protocol of casting, wound check and rehabilitation was followed. At last follow-up, patient was ambulating non-weightbearing with crutches (Fig. 8).

\section{DISCUSSION}

Achilles tendon is the strongest tendon of human body. ${ }^{9}$ It plays an important role of push off during the gait cycle and is also known to function as peripheral heart to aid in venous return from the lower extremities. It has a peculiar anatomy and the blood supply is derived primarily from the paratenon. Because of the mechanical stress and demands tendon ruptures are quite common. Also, it bears the brunt of many systemic disorders, like diabetes, SLE, alkaptonuria, autoimmune disorders and local intervention, such as steroid injection. Of the various reported causes, direct injuries have been seldom reported.

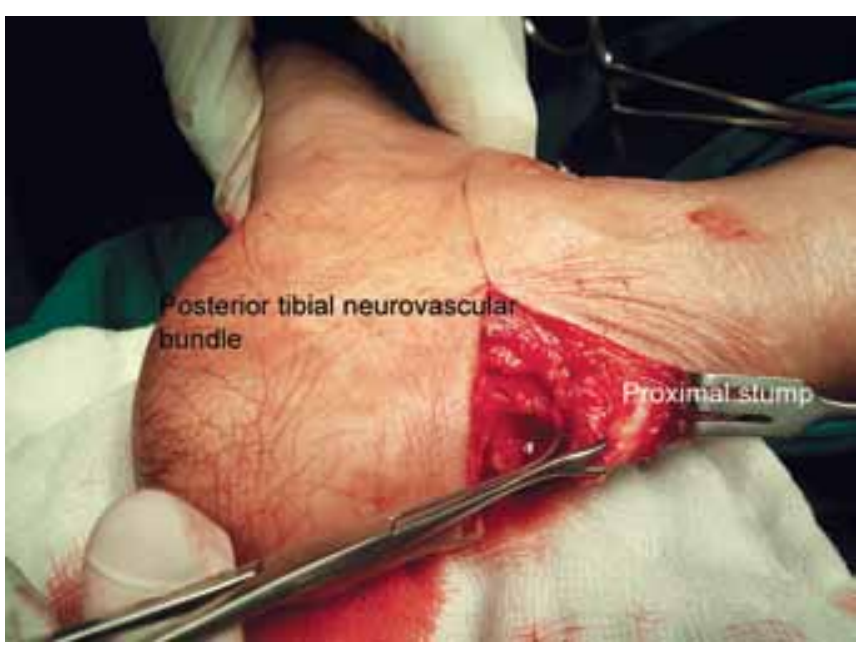

Fig. 7: Intraoperative picture showing the sharply incised ends of the TA tendon

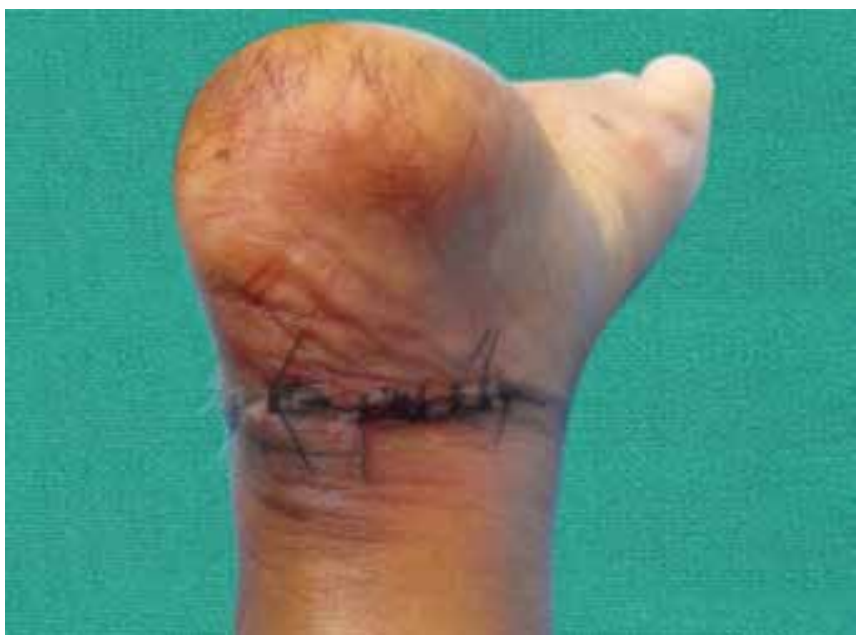

Fig. 8: Postopeartive picture after the wound closure with ankle in plantar flexion 


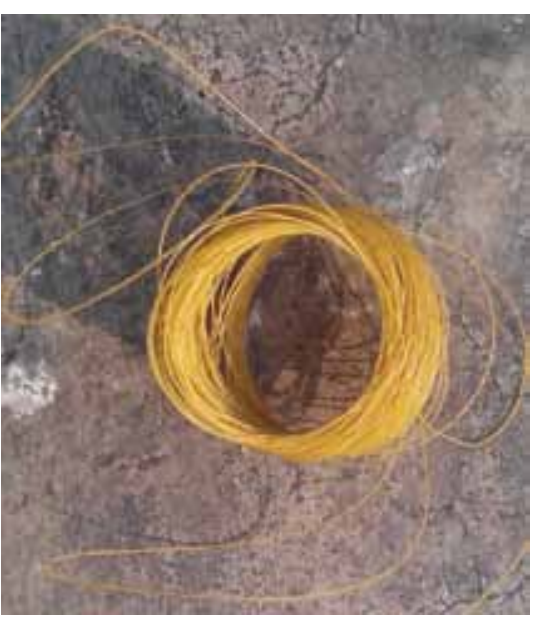

Fig. 9: Special type of kite string called as manja made of polypropylene thread colloquially called as Chinese manja (because it was chiefly imported from China)

Kite flying is among one of the popular games in children and adults in India, Pakistan, Bangladesh, Brazil. In India, 'Sankranti' is also called as festival of kites. In this game, kite is flied with a special thread called 'manja' (Fig. 9) which is made from cotton thread coated with powdered glass mixed in glue. Recently, a manja made of polypropylene thread colloquially called as Chinese manja (because it was imported from China) became very popular in view of increased strength of the same. It was later banned due to increased hazards, it posed to humans and animals in particular the birds. In this festival, a player rises to cut the string of opponent's kite thread while kite is in the air. As a result of this sharp thread many injuries are reported cervical cut throat injuries in two wheeler riders, ${ }^{1-4,6}$ fatal subcutaneous emphysema, ${ }^{2}$ electrical injuries ${ }^{1,5,7}$ falls. ${ }^{1}$ The severity of the injury depends on the speed of the vehicle and kite, which is directly related to wind speed. ${ }^{1}$ To best of our knowledge complete traumatic rupture of TA due to manja has so far not been reported.

Complete rupture of the Achilles tendon usually occurs in sports that require jumping, running and quick turns. ${ }^{8}$ Other causes of Achilles tendon rupture are overuse tendinopathy, inflammatory, autoimmune, genetically determined collagen abnormality, infectious diseases, neurological conditions, steroid local/systemic, hyperparathyroidism, ${ }^{10}$ alkaptonuria, ${ }_{1}^{11}$ fluoroquinolones, ${ }^{12}$ gout, ${ }^{13}$ calcification of tendon, ${ }^{14}$ Reiter's syndrome, ${ }^{15}$ sciatica. ${ }^{16}$

It is generally accepted that surgery should be performed on ruptured Achilles tendon in young, physically active patients for whom the diagnosis or the treatment of the rupture has been delayed, whereas the results of conservative treatment are an acceptable outcome in older patients with sedentary lifestyles. ${ }^{9}$
The aim of this article is to reinforce the hazards of manja and its variant Chinese manja. Also, it highlights that the appearance of the wound could be deceptive giving a false impression of benign looking wound which could be just a tip of iceberg. Underlying tendon and nerve injuries should always be ruled out in any type of wound. The late consequences of repair include increased morbidity, delayed recovery and loss of productive days. In case of acute traumas, it is important to follow the basics of thorough debridement relevant prophylaxis and a meticulous distal neurovascular examination.

\section{REFERENCES}

1. Ventura J, Hirano IES, Fraga IIGP. Glass-coated kites and cervical injuries: a serious threat to children and adults. Clinics 2011;66(5):923-925.

2. Tumram NK, Bardale RV, Dixit PG, Ambade VN. Fatal subcutaneous emphysema by manja: a deadly string. BMJ Case Rep 2013 Feb 13;2013.

3. Wankhede AG, Sariya DR. Manja-a dangerous thread. J Forensic Leg Med 2008 Apr;15(3):189-192.

4. Roberto MS, Paulo RC, John B, Gabriel CI, André AE. Epidemiology of powdered glass-coated kite line injuries: trauma victims admitted to the hospital in belo horizonte. Brazil Rev Bras Epidemio São Paulo 2012 Jun;15(2).

5. Tiwari VK, Sharma D. Kite-flying: a unique but dangerous mode of electrical injury in children. Burns 1999;25(6):537-539.

6. Neto JB, Ferreira GC, Filho AL, Fontes MO, Bomfim F, Abrantes WL. Kiting injuries: report of two cases and discussion. J Trauma 2000;48(2):310-311.

7. Wankhede AG, Sariya DR. An electrocution by metal kite line. Forensic Sci Int 2006;163(3):141-143.

8. Järvinen TA, Kannus P, Maffulli N, Khan KM. Achilles tendon disorders: etiology and epidemiology. Foot Ankle Clin 2005 Jun;10(2):255-266.

9. Järvinen TA, Kannus P, Paavola M, Järvinen TL, Józsa L, Järvinen M. Achilles tendon injuries. Curr Opin Rheumatol 2001 Mar;13(2):150-155.

10. Maffulli N. Current concepts review rupture of the achilles tendon. JBJS 1999 Jul;81A:7.

11. Kumar RVM, Rajasekaran S. Spontaneous tendon ruptures in alkaptonuria. J Bone Joint Surg 2003 Aug;85B(6):883-886.

12. Tsai WC. Fluoroquinolone-associated tendinopathy chang gung. Med J 2011 Sep-Oct;34(5):461-466.

13. Patrick GM, Peter DJ, Christopher JH, Anthony JS. Spontaneous rupture of the achilles tendon in a patient with gout. Annals of the Rheumatic Diseases 1981;40(5):416-418.

14. Wick MC, Rieger M. Rupture of a calcified achilles' tendon. N Engl J Med 2008;358:2618.

15. Azuma N, Kuno H, Imamura F, et al. A case of achilles tendon ruptures of refractory Reiter's syndrome. JPN J Clin Immunol 2008;31(2):113-118.

16. Maffulli N, Andrew SI, Michael GK, Francis S, Richard WP. Achilles tendon rupture and sciatica: a possible correlation. Br J Sports Med 1998;32(2):174-177. 